\title{
Cigarette smoking cessation increases plasma levels of several antioxidant micronutrients and improves resistance towards oxidative challenge
}

\author{
M. Cristina Polidori ${ }^{1 *}$, Patrizia Mecocci ${ }^{2}$, Wilhelm Stahl ${ }^{1}$ and Helmut Sies ${ }^{1}$ \\ ${ }^{1}$ Institute of Biochemistry and Molecular Biology I, Heinrich-Heine University, PO Box 101007, Düsseldorf, \\ D-40001, Germany \\ ${ }^{2}$ Institute of Gerontology and Geriatrics, Perugia University Hospital, Perugia, Italy
}

(Received 29 July 2002 - Revised 31 January 2003 - Accepted 17 March 2003)

\begin{abstract}
Cigarette smoking is associated epidemiologically with increased risk of cardiovascular diseases, but the pathophysiological mechanisms are still not fully understood. There is evidence that smoking is related to increased free radical production and antioxidant depletion, but the effects of smoking cessation on plasma concentrations of antioxidants and susceptibility to oxidative stress are largely unknown. Plasma levels of vitamins A, C, E, uric acid, total thiols, carotenoids (including lutein, zeaxanthin, $\beta$-cryptoxanthin, lycopene, $\alpha$ - and $\beta$-carotene) and malondialdehyde (MDA, a biomarker of lipid peroxidation) were measured in fifteen healthy, normolipidaemic subjects (seven males, eight females, $35 \cdot 2$ (SD 2.3) years) before and 4 weeks after smoking cessation. To determine plasma resistance towards oxidative challenge, plasma was incubated for up to $5 \mathrm{~h}$ with the peroxyl radical-generator 2,2'-azobis(2-amidinopropane) (AAPH); MDA and ascorbate levels were measured at various time points. The concentrations of all plasma antioxidants were lower before smoking cessation than afterwards; MDA levels were higher before than after termination of smoking. Upon AAPH exposure, the consumption of plasma ascorbate and the production of MDA occurred at a significantly faster rate before smoking cessation as compared with afterwards. Cigarette smoking cessation is followed by a marked increase in plasma antioxidant concentrations and substantially improves plasma resistance towards oxidative challenge. Given the importance of cigarette smoking as a risk factor for cardiovascular diseases and the pathophysiological role played by oxidative stress in these illnesses, quitting smoking represents an irreplaceable preventive strategy against tobacco-induced oxidative stress and vascular damage.
\end{abstract}

Smoking: Antioxidants: Micronutrients: Oxidative stress

Cigarette smoking is associated epidemiologically with a high risk for various types of chronic illnesses including vascular disease (McNamara \& FitzGerald, 2001). The underlying mechanisms of smoke-related severe damage to tissues and organs are still not completely understood; $\mathrm{O}-$ and N-derived free radicals are thought to play a major pathophysiological role (Pryor \& Stone, 1993).

Significantly lower plasma carotenoid and vitamin $\mathrm{C}$ levels have been shown in Scottish male smokers as compared with non-smokers (Ross et al. 1995). Male smokers aged 18-26 years showed significantly lower serum $\beta$-carotene levels and significantly higher serum lipid hydroperoxide levels as compared with age-matched non-smokers (al Senaidy et al. 1997).

It is known that smoking cessation leads to a significant increase of plasma vitamin C (Lykkesfeldt et al. 1996) and of glutathione concentrations (Lane et al. 1996), but its effects on plasma concentrations of several antioxidants and susceptibility to oxidative stress are unknown.

The aims of the present study were, therefore, to evaluate the effects of smoking cessation on the plasma concentrations of a broad spectrum of water-soluble and lipophilic compounds with antioxidant properties and of malondialdehyde (MDA), a marker of free radical-induced lipid peroxidation, as well as on the susceptibility of plasma to oxidative stress.

\section{Subjects and methods}

The investigation conforms to the principles outlined in the Declaration of Helsinki. Fifteen healthy volunteers (seven males, eight females, $35 \cdot 2$ (SD 2.3) years) smoking $\geq$ seven cigarettes/d for at least 6 years each provided $20 \mathrm{ml}$ blood after an overnight fast. The blood was placed into two $10 \mathrm{ml}$ tubes containing heparin at 08.00 hours. The subjects gave informed consent. They were allowed to smoke on the day of the blood drawing; they did not smoke on the next day or afterwards. After 04 weeks, two other blood samples were obtained from the same subjects at 08.00 hours after an overnight fast. Blood was immediately centrifuged and plasma $(8 \mathrm{ml})$ separated for the assays. Subjects did not change dietary 
or exercise habits during the time preceding the second blood drawing, as assessed by a standardized interview (performed by an independent researcher blinded to the measurements), which included the administration of a food-frequency questionnaire (Winkler \& Döring, 1998). Before smoking cessation, volunteers were asked to recall their average intake of food items in the following six frequency categories: almost daily; several times per week; about once weekly; several times per month; once monthly or less; never. On the day of the first blood withdrawal, subjects were counselled to follow the same dietary pattern for the next 4 weeks. The food-frequency questionnaire was re-administered weekly. There were no missing data for the complete questionnaires or for single items. Exclusion criteria were constituted by BMI $<18.5$ or $>30 \mathrm{~kg} / \mathrm{m}^{2}$, malnutrition, vitamin consumption, diabetes, hypertension, any organ and/or psychiatric illness and abnormalities of lipid profile as assessed by total cholesterol $\geq 6.2 \mathrm{mmol} / \mathrm{l}$, LDL-cholesterol $\geq 4.1 \mathrm{mmol} / \mathrm{l}$, and triacylglycerol $\geq 3.4 \mathrm{mmol} / \mathrm{l}$.

Of the $8 \mathrm{ml}$ plasma separated from the blood of volunteers before and after smoking cessation, $1 \mathrm{ml}$ was used for measurements of cotinine concentrations and of antioxidant and MDA concentrations. Plasma cotinine was determined by radioimmunoassay as previously described (Langone et al. 1973). Vitamin C and uric acid were detected by HPLC with electrochemical detection as previously described (Frei et al. 1989). Carotenoids including lutein, zeaxanthin, $\beta$-cryptoxanthin, total lycopene, $\alpha$-carotene and $\beta$-carotene were measured by HPLC with u.v. and visible detection according to Stahl et al. (1993). A second u.v. and visible detector was connected in series and set at $325 \mathrm{~nm}$ and at $292 \mathrm{~nm}$ for quantification of retinol (vitamin A), and $\alpha$-tocopherol and $\gamma$-tocopherol, respectively. Total plasma thiol groups were measured spectrophotometrically (Motchnik et al. 1994). MDA concentrations were measured by HPLC with fluorescence detection (excitation $513 \mathrm{~nm}$, emission $550 \mathrm{~nm}$ ) (Suttnar et al. 1997).

Plasma $(7 \mathrm{ml})$ was incubated for $5 \mathrm{~min}$ at $37^{\circ} \mathrm{C}$, and $750 \mathrm{~mm}-2,2^{\prime}$-azobis(2-amidinopropane) (AAPH) $(500 \mu \mathrm{l})$ was added (final concentration, $50 \mathrm{~mm}$ ). Immediately after the addition of AAPH (time 0), and every $30 \mathrm{~min}$ up to $5 \mathrm{~h}$, samples were taken for the determination of vitamin $\mathrm{C}$ and MDA concentrations.

All data are presented as mean values and standard deviations. Statistical analysis was performed with the program SPSS 10.0 (SPSS Inc., Chicago, IL). Nonparametric Wilcoxon signed ranking test was used for comparisons between values before and after smoking cessation. A generalized linear model for repeated measures was used for comparisons between AAPH-induced vitamin $\mathrm{C}$ consumption and MDA production over time before and after smoking cessation. Significance was accepted if the null hypothesis was rejected at the $P<0.05$ level.

\section{Results and discussion}

All subjects studied showed decreased concentrations of plasma cotinine after smoking cessation (1.6 (SD 0.7) v. $0.7(\mathrm{SD} 0.2) \mathrm{ng} / \mathrm{ml} ; P<0.04)$. Before smoking cessation, all subjects showed lower plasma antioxidant levels than afterwards. Differences reached statistical significance for vitamin $\mathrm{C}, \alpha$-tocopherol, $\gamma$-tocopherol, vitamin $\mathrm{A}$, lutein, zeaxanthin, lycopene and $\beta$-carotene (Table 1). There were no significant differences between values in male and female subjects before and after smoking cessation. In agreement with our previous results (Polidori et al. $2001 b$ ), as well as with the study by Lykkesfeldt et al. (1996), who showed that plasma vitamin C concentrations increased from 45.4 (SD 20.2) $\mu \mathrm{M}$ before smoking cessation to 56.0 (SD 24.2) $\mu \mathrm{M}$ after smoking cessation in 100 subjects, an increase in vitamin $\mathrm{C}$ plasma levels from $50 \cdot 0$ (SD 11.8) to 62.9 (SD 13.9) $\mu \mathrm{M}$ was observed in the smoking quitters included in the present study. Eiserich et al. (1995) reported that plasma exposure to gas-phase cigarette smoke caused depletion of carotenoids, vitamin $\mathrm{C}$, uric acid and $\alpha$-tocopherol, and induction of lipid peroxidation in vitro. In vivo studies strongly support the relationship between smoking habit and depletion of a number of dietary components with various degrees of antioxidant capacity, including vitamin $\mathrm{C}$, $\alpha$-tocopherol and carotenoids (Eiserich et al. 1995; Ross et al. 1995; al Senaidy et al. 1997). It is conceivable that, in a condition of cigarette smoke-related oxidative stress, compounds with stronger antioxidant capacity such as ascorbate, the most potent water-soluble antioxidant in the organism whose levels have been shown to be negatively associated to the severity of both ischaemic (Cherubini et al. 2000) and haemorrhagic (Polidori et al. 2001a) stroke, are consumed first.

In the present study, plasma levels of lycopene and lutein, among other carotenoids, also markedly increased after smoking cessation (Table 1). Both of these carotenoids possess an effective free radical-scavenging ability, and might have a synergistic antioxidant effect; their plasma levels have been shown to be inversely correlated

Table 1. Plasma concentrations $(\mu \mathrm{M})$ of vitamin $C$, uric acid, total thiols, $\alpha$-tocopherol, $\gamma$-tocopherol, vitamin A, lutein, zeaxanthin, $\beta$-cryptoxanthin, lycopene, $\alpha$-carotene, $\beta$-carotene, and malondialdehyde before and after smoking cessation and relative $P$ values $†$ (Mean values and standard deviations)

\begin{tabular}{|c|c|c|c|c|c|}
\hline \multirow[b]{2}{*}{ Compound } & \multicolumn{2}{|c|}{$\begin{array}{l}\text { Before smoking } \\
\text { cessation }\end{array}$} & \multicolumn{2}{|c|}{$\begin{array}{l}\text { After smoking } \\
\text { cessation }\end{array}$} & \multirow[b]{2}{*}{$P$ value } \\
\hline & Mean & SD & Mean & SD & \\
\hline Vitamin C & $50 \cdot 0$ & $11 \cdot 8$ & $62 \cdot 9^{\star}$ & $13 \cdot 9$ & 0.0007 \\
\hline Uric acid & 303 & 26 & 306 & 50 & 0.394 \\
\hline Total thiols & 336 & 62 & 339 & 65 & 0.108 \\
\hline$\alpha$-Tocopherol & 23.5 & 3.5 & $26 \cdot 2^{\star}$ & $3 \cdot 2$ & 0.0010 \\
\hline$\gamma$-Tocopherol & $2 \cdot 0$ & 0.4 & $2 \cdot 2^{\star}$ & 0.4 & 0.0007 \\
\hline Vitamin A & 2.4 & 0.6 & $2 \cdot 8^{*}$ & 0.6 & 0.0007 \\
\hline Lutein & 0.34 & 0.1 & $0.45^{\star}$ & 0.1 & 0.0010 \\
\hline Zeaxanthin & 0.09 & 0.03 & $0.10^{*}$ & 0.02 & 0.018 \\
\hline$\beta$-Cryptoxanthin & 0.10 & 0.02 & 0.11 & 0.02 & 0.077 \\
\hline Lycopene & 0.51 & 0.1 & $0.72^{\star}$ & 0.1 & 0.0007 \\
\hline$\alpha$-Carotene & 0.02 & 0.01 & 0.03 & 0.01 & 0.388 \\
\hline$\beta$-Carotene & 0.18 & 0.06 & $0.21^{*}$ & 0.07 & 0.0010 \\
\hline Malondialdehyde & 0.81 & 0.12 & $0.58^{*}$ & 0.15 & 0.0007 \\
\hline
\end{tabular}

* Mean value was significantly different from the before smoking cessation value $(P<0.05$; non-parametric Wilcoxon signed ranking test).

†For details of procedures, see p. 148. 
with the severity of ischaemic stroke (Polidori et al. 2002a) and congestive heart failure (Polidori et al. 2002b). Simultaneously, a significant decrease in plasma MDA concentrations in smoking quitters was observed; interestingly, plasma levels of this biomarker of free radical-induced lipid peroxidation are directly correlated with the severity of ischaemic stroke (Polidori et al. 2002a) and congestive heart failure (Polidori et al. 2002b).

Exposure of plasma to AAPH, which produces peroxyl radicals at a known constant rate, caused more rapid vitamin $\mathrm{C}$ consumption $(P<0.001)$ and MDA production $(P<0.001)$ in smokers as compared with quitters (Fig. 1). Most importantly, plasma vitamin $\mathrm{C}$ was completely consumed after 70 (SD 15) min before smoking cessation and 140 (SD 15) min after smoking cessation $(P<0 \cdot 001)$. One possible reason for the lower rate of ascorbate oxidation following smoking cessation might be the influence of biomolecules with antioxidant activity other than vitamin $\mathrm{C}$ (for instance, bilirubin) on the reaction of ascorbate with AAPH-derived peroxyl radicals. MDA plasma levels started to increase after vitamin $\mathrm{C}$ was completely consumed both before and after smoking cessation. Our findings are highly consistent with previous studies (Frei, 1991) showing that only when ascorbate, but no other antioxidants, is completely consumed after plasma AAPH exposure does lipid peroxidation start (Frei, 1991). In later experiments, it was shown that plasma devoid of ascorbate, but no other antioxidants, and exposed to AAPH is extremely vulnerable to lipid peroxidation, and that this protection exerted by ascorbate against lipid peroxidative damage is highly effective even in the presence of bleomycin-detectable Fe (Berger et al. 1997).

The results shown in the present paper demonstrate that smoking cessation is accompanied by the elevation of several critical components of the antioxidant defence system of the organism. The finding that this elevation occurs in as little time as 4 weeks is in agreement with the recent observation that smoking a single cigarette rapidly lowers nitrate, nitrite and plasma levels of ascorbate, urate and cysteine (Tsuchiya et al. 2002).

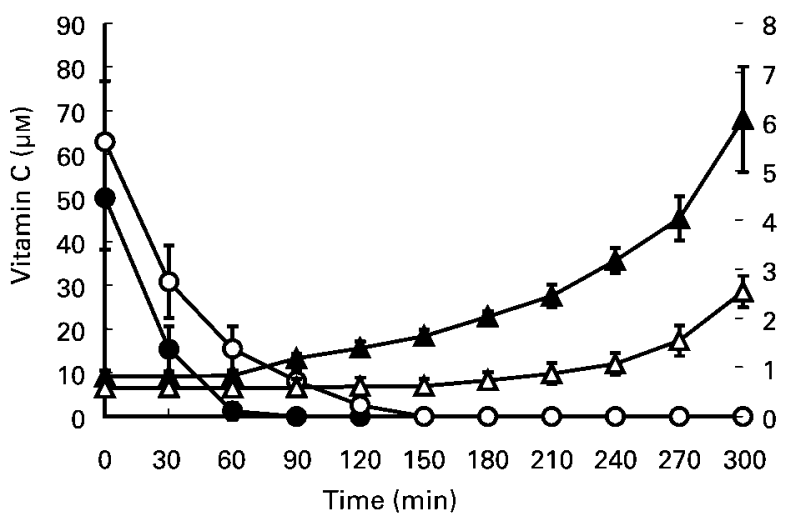

Fig. 1. Time course of plasma vitamin $C(\bullet, O)$ and malondialdehyde (MDA; $\boldsymbol{\Lambda}, \triangle$ ) concentrations after 2,2'-azobis(2-amidinopropane) addition. $(\boldsymbol{O}, \boldsymbol{\Delta})$, Before smoking cessation; $(\bigcirc, \Delta)$, after smoking cessation. Mean values are shown for plasma from seven male and eight female volunteers, with standard deviations being represented by vertical bars.
In our study, quitters also showed lower plasma lipid peroxidation than before stopping smoking, as well as a decrease in plasma susceptibility to vitamin $\mathrm{C}$ depletion and MDA formation after exposing plasma to a flux of aqueous peroxyl radicals generated at a known constant rate. Lipid peroxidation and antioxidant depletion in smokers may be significant contributors to biomolecular endothelial vascular damage. Although antioxidant supplementation might be helpful in preventing or attenuating some cigarette smoke-related adverse effects (Reilly et al. 1996), quitting smoking remains a secure means by which smokers can prevent tobacco-induced oxidative stress and cardiovascular diseases.

\section{Acknowledgements}

M. C. P. is a European Union Marie-Curie Fellow for the programme 'Quality of Life and Management of Living Resources' and project entitled 'Nutritional health-sustaining factors and determinants of healthy aging: oxidative stress-related biomarkers of successful aging and agerelated diseases'. H. S. is a Fellow of the National Foundation for Cancer Research, Bethesda, MD.

\section{References}

al Senaidy AM, al Zahrany YA \& al Faqeeh MB (1997) Effects of smoking on serum levels of lipid peroxides and essential fatsoluble antioxidants. Nutr Health 12, 55-65.

Berger TM, Polidori MC, Dabbagh A, et al. (1997) Antioxidant activity of vitamin $\mathrm{C}$ in iron-overloaded human plasma. J Biol Chem 272, 15656-15660.

Cherubini A, Polidori MC, Bregnocchi M, et al. (2000) Antioxidant profile and early outcome in stroke. Stroke 31, 2295-2300.

Eiserich JP, van der Vliet A, Handelman GJ, Halliwell B \& Cross CE (1995) Dietary antioxidants and cigarette smokeinduced biomolecular damage: a complex interaction. Am J Clin Nutr 62, 1490S-1500S.

Frei B (1991) Ascorbic acid protects lipids in human plasma and low-density lipoprotein against oxidative damage. Am J Clin Nutr 54, 1113S-1118S.

Frei B, England L \& Ames BN (1989) Ascorbate is an outstanding antioxidant in human blood plasma. Proc Natl Acad Sci USA 86, 6377-6381.

Lane JD, Opara EC, Rose JE \& Behm F (1996) Quitting smoking raises whole blood glutathione. Physiol Behav 60 , $1379-1381$.

Langone J, Gjika HB \& Van Vunakis H (1973) Nicotine and its metabolites: Radioimmunoassay for nicotine and cotinine. Biochemistry 12, 5025-5030.

Lykkesfeldt J, Priemé H, Loft S \& Poulsen HE (1996) Effect of smoking cessation on plasma ascorbic acid concentration. Br Med J 313, 91.

McNamara P \& FitzGerald GA (2001) Smoking-induced vascular disease. A new twist on an old theme. Circ Res 89, 563-565.

Motchnik PA, Frei B \& Ames BN (1994) Measurement of antioxidants in human blood plasma. Methods Enzymol 234, 269-279.

Polidori MC, Cherubini A, Stahl W, Senin U, Sies H \& Mecocci P (2002a) Plasma carotenoid and malondialdehyde levels in ischemic stroke patients: relationship to early outcome. Free Radic Res 36, 265-268. 
Polidori MC, Mecocci P \& Frei B (2001a) Plasma vitamin C levels are decreased and correlated with brain damage in patients with intracranial hemorrhage or head trauma. Stroke 32, 898-902.

Polidori MC, Savino K, Alunni G, et al. (2002b) Plasma lipophilic antioxidants and malondialdehyde in congestive heart failure patients: relationship to disease severity. Free Radic Biol Med 32, 148-152.

Polidori MC, Stahl W, Eichler O, Niestroj I \& Sies H (2001b) Profiles of antioxidants in human plasma. Free Radic Biol Med 30, 456-462.

Pryor WA \& Stone K (1993) Oxidants in cigarette smoke. Radicals, hydrogen peroxide, peroxynitrate, and peroxynitrite. Ann NY Acad Sci 686, 12-27.

Reilly M, Delanty N, Lawson JA \& FitzGerald GA (1996) Modulation of oxidant stress in vivo in chronic cigarette smokers. Circulation 94, 19-25.
Ross MA, Crosley LK, Brown KM, et al. (1995) Plasma concentrations of carotenoids and antioxidant vitamins in Scottish males: influences of smoking. Eur J Clin Nutr 49, $861-865$.

Stahl W, Sundquist AR, Hanusch M, Schwarz W \& Sies H (1993) Separation of $\beta$-carotene and lycopene geometrical isomers in biological samples. Clin Chem 39, 810-814.

Suttnar J, Cermak J \& Dyr JE (1997) Solid-phase extraction in malondialdehyde analysis. Analytical Biochemistry 249, 20-23.

Tsuchiya M, Asada A, Kasahara E, Sato EF, Shindo M \& Inoue M (2002) Smoking a single cigarette rapidly reduces combined concentrations of nitrate and nitrite and concentrations of antioxidants in plasma. Circulation 105, 1155-1157.

Winkler G \& Döring A (1998) Validation of a short qualitative frequency list used in several German large scale surveys. Eur J Nutr 37, 234-241. 